Review

\title{
Emerging Significance and Therapeutic Potential of Extracellular vesicles
}

\author{
Ruhua Luo, Mengmeng Liu, Tiantian Tan, Qian Yang, Yue Wang, Lianhui Men, Liping Zhao, Honghua \\ Zhang, Shuling Wang ${ }^{\Perp}$, Tian Xie ${ }^{凶}$, Qingchang Tian ${ }^{\bowtie}$ \\ 1. College of Pharmacy, School of Medicine, Hangzhou Normal University, Hangzhou, Zhejiang 311121, China. \\ 2. Key Laboratory of Elemene Class Anti-Cancer Chinese Medicines; Engineering Laboratory of Development and Application of Traditional Chinese \\ Medicines; Collaborative Innovation Center of Traditional Chinese Medicines of Zhejiang Province, Hangzhou Normal University, Hangzhou, Zhejiang \\ 311121, China. \\ $\triangle$ Corresponding authors: Shuling Wang (wsling222@163.com), Tian Xie (xbs@hznu.edu.cn), Qingchang Tian (tianqc@hznu.edu.cn);
}

(C) The author(s). This is an open access article distributed under the terms of the Creative Commons Attribution License (https://creativecommons.org/licenses/by/4.0/). See http://ivyspring.com/terms for full terms and conditions.

Received: 2021.02.10; Accepted: 2021.05.27; Published: 2021.06.16

\begin{abstract}
Extracellular vesicles (EVs), are membrane-bound vesicles that have many advantages over traditional nanocarriers for drug and gene delivery. Evidence from recent studies indicate that EVs have therapeutic capability with chemical or biological modification. Tumor-derived exosomes (TEXs) were used as a new type of antigens or tumor vaccines in anti-tumor immunotherapy. With superior characteristics, modified EVs were applied to loaded and delivered synthetic drugs, silencing RNA, and microRNA for treatment. Different surface functionalization strategies have been proposed to improve the therapeutic functions of EVs. Appropriately modified EVs for disease intervention provide new avenues for effective clinical treatment strategies. Therefore, this review aimed at elucidating the therapeutic functions of EVs to generate new ideas for treatment and to unlock their hidden potential in translational medicine.
\end{abstract}

Key words: Extracellular vesicles; Exosomes; Tumor-derived exosomes; Delivery vehicles;

\section{Introduction}

Cancer is a global human health problem that is associated with severe pain and heavy economic burdens to patients and their families [1]. Studies on EVs have elucidated on new strategies for cancer diagnosis and treatment. In recent years, nanomaterials for drug delivery have played an important role in the treatment of cancer [2]. However, they are associated with certain limitations. Therefore, studies are evaluating the value of EVs in the development of a comprehensive drug delivery system and as a circulating biomarker in cancer diagnosis [3]. The International Society for Extracellular Vesicles (ISEV) recognized the potential of EVs as treatments for cancer. EVs or exosome nanovesicles (NVs), which combine the characteristics of cells and nanocarriers, are clinically used in tumor diagnosis [4], and targeted anticancer therapies, such as breast [5], pancreatic [6], lung [7], and liver cancers [8].

In guidelines released by the ISEV, depending on their biogenesis and generation pathways, EVs can be broadly classified into three subpopulations: exosomes (EXOs, 30 to $200 \mathrm{~nm}$ in size), microvesicles (MVs, 200-1000 nm), and apoptotic bodies (ABs, larger than $1000 \mathrm{~nm}$ ) [9]. EVs are extracellular nanovesicles secreted by various kinds of cells including dendritic cells (DC) [10], mesenchymal stem cells (MSCs) [11], neural cells [12], epithelial cells [13], and a variety of tumor cells [14]. Interestingly, EVs are also distributed in serum, urine, saliva, or any other body fluids [15, 16]. They contain and tranfer diversified bioactive molecules include lipids, nucleic acids, and various proteins inside or on the surface of EVs, such as receptors, enzymes, transcription factors, and extracellular matrix proteins into adjacent or distant cells through the systemic circulation, participating in intracellular and intercellular communication, and regulate host tumor cell interactions [17]. As a general rule, of the targeting and fusion proteins present on the surface of EVs, the most abundant are integrins and Tetraspanins. The 
members of the tetraspanin family, such as CD9, CD63, CD81 and CD82, regulate intercellular signal transduction [18, 19]. As EVs contain biomolecules active from maternal cells, they can regulate function, fate, and shape of target cells, participating in different pathological and physiological conditions $[20,21]$. Conserved proteins participate in cytoskeleton formation ( $\beta$-actin, myosin and tubulins), metabolism (glyceraldehyde 3-phosphate dehydrogenase) and protein folding (Hsp70) [22, 23]. Cell-type-specific proteins such as notch ligands, $\beta$-Catenin, Wnt as well as intercellular cell signaling mediators mainly involved in cell signaling pathways such as TNF- $\alpha$, TGF- $\beta$, and IL-1 $\beta$ [24]. Of course, EVs also contain nucleic acids, including mRNA, miRNA, DNA, and mitochondrial DNA (mtDNA). These nucleic acids are mainly involved in inflammation and as diagnostic biomarkers in tumors [25, 26]. In recent years, scientists have paid more attention to EVs due to their key roles in biological systems.

Exosomal liposome structures allow the loading of various drugs, which enhances drug delivery to specific targets. EVs, as carriers, can transport their cargos to particularly intracellular locations in a target-specific manner across the plasma membrane. As natural nucleic acid and protein carriers, they have been used as vectors for targeted delivery of these molecules [27, 28]. Based on their endogenous performances and multifunctional properties, EVs have a clinical potential for the development of efficient therapeutic options for cancer.

Evidence from recent studies indicate that EVs have therapeutic potential in tumors [29], neurological diseases [30] and immune diseases [31]. For instance, exosomes are not only used as carriers for tumor treatment drugs [32], but also as tumor immunotherapy, especially for those extracted from tumor cells robustly eliciting anti-tumor immune responses [33,34]. Given their low immunogenicity and high biocompatibility, exosomes can stably stay in the circulatory system for longer periods. Targeted administration of exosomes carrying drugs to tumor lesions can double the anti-tumor effect of such drugs. When used as carriers, EVs not only provide intrinsic immunomodulatory activity, but also have many advantages such extended circulation half-life, high biocompatibility, transfection efficiency, low immunogenicity and minimal reversion to virulence, over traditional nanocarriers for drug and gene delivery [34]. Hence, EVs may provide opportunities to enhance or broaden the innate therapeutic capability with chemical or biological modification.

\section{EVs in cancer immunotherapy}

Immunotherapy has shown promising prospects in the treatment of cancers, and EVs are currently applied in tumor immunotherapy. EVs have several advantages and appear to be a highlight of new pattern for cancer immunotherapy at present [35]. The use of EVs as a new type of antigens in anti-tumor immunotherapy has raise some concerns. By reactivating the patient's immune system, EVs exploit autoimmune cells, especially CD8+ T cells, to generate anti-tumor responses [36]. Although the potential of these therapies is widely known, there is still significant room for improvement. Associated immune checkpoint therapies tend to be ineffective and severe autoimmunity could also occur. Many tumors, due to genetic, biological and other factors make it unlikely that some patients will respond to these therapies. To overcome some of these obstacles, innovative approaches with lower toxicity and providing more frequent and durable response are needed. One such treatment is the use of nanoparticles, particularly EVs. Research has shown that TEXs potentiate PD-L1 function and suppress immune response [37]. In addition, in patients with melanoma receiving PD-1 blockade, PD-L1 levels in exosomes are correlated with tumor burden and treatment response. Although it is unclear whether the exosomal PD-L1 is directly related the failure of anti-PD-1 therapies, PD-L1-containing exosomes may be regulators and biomarkers of resistance to treatment.

Surface engineered EVs can actively participate in tumor immunotherapy. CD47, a "don't eat me" signal, limits the ability of macrophages to engulf tumor cells by binding to SIRPa [38]. Therefore, the exosomes carrying SIRPa variants may serve as immune checkpoint blockers, thereby antagonize the interaction between CD47 and SIRP. This will enhance tumor phagocytosis and exert effective anti-tumor $\mathrm{T}$ cell response [39]. Although the immunotherapeutic effects of dendritic cell vaccine have been reported, it is still unclear how tumor-associated exosome-based dendritic cell (DC) vaccine-based anti-tumor immunity can be induced to achieve antitumor effects [40]. Studies have shown that dendritic cell-derived exosomes (DEX) can regulate the immune responses to cancer [41]. DEXs stabilizes vesicles, and are not easily degraded or inactivated. Research on DEXs has shown that DEX is rich in membranous proteins such as major histocompatibility complexes class I (MHC class I), MHC class II molecules, CD63, CD81, and integrin, and has a strong immune activation effect [42]. The use of EVs as tumor vaccines has shown promising anticancer effects in vivo, and results from clinical trials on EVs have been feasible. A novel DEX vaccine with antigens and matured with either the TLR-3 ligand induced robust activation of 
melanoma-specific CD8(+) T cells and the recruitment of cytotoxic CD8(+) T cells, NK and NK-T cells to the tumor site, resulted in significantly reduced tumor growth and enhanced survival [43]. A phase I clinical trials to test DEXs loaded MAGE tumor antigens in patients with non-small cell lung cancer (NSCLC) showed that some patients experienced long term stability of disease and activation of immune effectors [44]. A phase II clinical trial testing the clinical benefit of DEX loaded with MHC class I- and class II-restricted cancer antigens as maintenance immunotherapy confirmed the capacity of DEX to boost the NK cell arm of antitumor immunity in patients with advanced NSCLC [45]. DEX maintain the key functions of DCs in their ability to present tumor-associated antigens (TAAs) and to activate TAA-specific immune responses [46]. To improve immunogenicity, exosomal antigen-adjuvant co-delivery systems have been engineered for cancer immunotherapy. Exosomes derived from genetically engineered tumor cells containing endogenous tumor antigens and CpG DNA with immunostimulatory have been found to confer anti-tumor effects [47]. Therefore, blocking the biogenesis and release of TEX seems to be a potential anti-tumor strategy. GW4869, an inhibitor of nSMase2, has been found to block ceramide synthesis. Treatment with GW4869 reduced lung metastasis in tumor-bearing mice. A combination of GW4869 with cisplatin and gefitinib provided strong antitumor effect [48].

\section{EVs as drug carriers in cancer}

Exploitation of EVs in anti-tumor research has gained momentum in recent years, and many studies have evaluated EVs -related antitumor effects. Compared with EVs, delivery of drug formulations such as liposomes [49], micelles [50], and microcapsules [51] using traditional carriers is limited in clinical application, due to immune rejection, low drug loading, and poor targeting. EVs, as carriers, do not elicit immune responses in the bloodstream like other nanoparticle formulations [52]. Given their superior characteristics to natural or synthetic polymers and liposomes, many studies (Table 1) have reported that synthetic drugs, silencing RNA, and microRNA can be loaded into modified EVs and delivered into tumors yielding good results.

The most common treatments for malignant tumors are chemotherapy, radiation surgery, or combination therapy. Cancer targeted therapy is an emerging treatment approach in anti-tumor therapy. However, the success of targeted therapy requires a drug delivery carrier with low immunogenicity and low toxicity. Doxorubicin (DOX) was loaded into exosomes through electroporation, and delivered to breast cancer tumor cells by engineered exosomes leading to effective targeting [53]. The main advantage of using exosomes to carry biological molecules over other nanoparticles is that they possess natural ability to carry bio-related molecules such as nucleic acid drugs and can activate the immune system. Ohno et al. modified exosomes with a GE11 peptide that specifically binds to epidermal growth factor receptor (EGFR) and was loaded with let-7a miRNA, a regulator that reduced cell division and altered the cell cycle [54]. A synthetic multivalent antibodies retargeted exosome (SMART-Exosome) was designed to cross-link tumor cells with $\mathrm{T}$ cells and induce a strong immune response which effectively killed tumor cells in vivo and in vitro [55]. Although numerous drugs have been designed for prevention of cancer progression and suppress tumor development, of the efficacy of such drugs is limited by the low bioavailability and high toxicity. A highly biocompatible tumor cell-targeted delivery system has been designed to deliver imperialine (a less toxic anti-cancer agent) into NSCLC cells using exosome-like vesicles (ELVs) [56]. Given the high expression of alpha 3 beta 1 on NSCLC cells, they modified integrin alpha 3 beta 1-binding octapeptide cNGQGEQc to create an ELV platform for targeting tumor. This platform not only significantly improved accumulation and retention of imperialine in the tumor, but also exhibited extremely low systemic toxicity in vitro and in vivo. Natural compounds such as anthocyanins (Anthos) found in berries are limited by low permeability and oral bioavailability. Munagala et al. delivered exosomes loaded with anthocyanins from raw milk to mice with lung cancer [52]. They reported that exosomes provided an effective alternative for oral delivery of Anthos for efficient systemic delivery and robust bioavailability. For nervous system tumors, the blood-brain barrier (BBB) limits the entry of therapeutic drugs into the brain. Yang et al. tried to further their zebrafish studies pass through loading siRNA in the exosomes [57].

\section{EVs for gene therapy}

Donor cells have also been engineered to isolate qualified exosomes that contain the gene or drug of interest [58]. Exosomes are secreted from engineered cells through the endosomal pathway, and this pathway have been hijacked with viruses and used for superior delivery of RNA in vivo [59]. Many studies have exploited the genetic material naturally carried by foreign bodies, and many cancer-based studies have investigated exosomes using microRNAs. Thus far, exosome-derived microRNAs, through target gene transcriptional repression, have 
the demonstrated ability to induce cell migration, inflammation, immune responses, angiogenesis, invasion, pre-metastatic niche formation and metastasis (Figure 1). Masaki et al. found that miR-199a-3p-Exo suppressed c-Met expression, a direct target of miR-199a-3p, leading to the inhibition of cell proliferation and invasion [60]. The engineered exosomes were utilized to simultaneously deliver the anticancer drug 5-Fu and the Mir-21 inhibitor oligonucleotide (Mir-21I) to cancer cells expressing human epidermal growth factor receptor-2 (HER2) [61]. This effectively reversed drug resistance in 5-FU-resistant colon cancer cells and significantly enhanced cytotoxicity. These studies highlight the potential of exosomes encapsulated with tumor suppressor miRNAs in the treatment of cancer.

Table 1. Overview of Cancer Type, Exosomal Cargo, Loading method and Source of EVs Discussed in This Review.

\begin{tabular}{|c|c|c|c|c|c|}
\hline Cancer type & Drug & Source of exosome & Loading method & Outcome & Ref. \\
\hline \multirow[t]{13}{*}{ Breast cancer } & Paclitaxel; Doxorubicin & Macrophage cells & Sonication & Inhibition of tumor growth & [66] \\
\hline & Doxorubicin; miR159 & THP-1 cells & Incubation & Silenced the TCF-7 gene & {$[67]$} \\
\hline & Taxol & Human mesenchymal stroma/stem-like cell & Incubation & $\begin{array}{l}\text { Inhibition of tumor metastases and target } \\
\text { specificity }\end{array}$ & [68] \\
\hline & siRNA & $\begin{array}{l}\text { HEK } 293 \text { with surface modification by } \\
\text { LAMP2b-DARPin G3 chimeric gene }\end{array}$ & Transduction & $\begin{array}{l}\text { Target specificity; TPD52 gene expression is } \\
\text { downregulated }\end{array}$ & [69] \\
\hline & Doxorubicin & Breast cancer cell line and mouse ovarian cells & Electroporation & Inhibition of tumor proliferation & {$[70]$} \\
\hline & Doxorubicin & Mouse immature dendritic cells & Electroporation & Inhibition of tumor proliferation & [53] \\
\hline & Erastin & HFL-1 cell & Sonication & $\begin{array}{l}\text { Inhibition of tumor proliferation and } \\
\text { metastases }\end{array}$ & [71] \\
\hline & Doxorubicin & J774A.1 cell & extrusion & Increased target specificity & [72] \\
\hline & Curcumin & Bovine milk & incubation & Inhibition of tumor growth & [73] \\
\hline & Paclitaxel & Macrophage cells & $\begin{array}{l}\text { Incubation; } \\
\text { electroporation; } \\
\text { sonication }\end{array}$ & Inhibition of tumor growth and metastases & [74] \\
\hline & Paclitaxel & Raw bovine milk & Incubation & Inhibition of tumor growth & [75] \\
\hline & Berry Anthos & Raw bovine milk & Incubation & Inhibition of tumor proliferation & {$[52]$} \\
\hline & Doxorubicin & J774A.1 cell & Electroporation & Promote apoptosis and silence target genes & {$[72]$} \\
\hline \multirow[t]{5}{*}{ Lung cancer } & Withaferin A & Bovine milk & Incubation & Reduced tumor growth & [76] \\
\hline & Curcumin & Bovine milk & Incubation & Reduced tumor growth & [77] \\
\hline & Celastrol & Raw bovine milk & Incubation & $\begin{array}{l}\text { Increased drug efficacy and inhibition of } \\
\text { tumor growth }\end{array}$ & [78] \\
\hline & Paclitaxel & Macrophage cell & Sonication & $\begin{array}{l}\text { Increased target specificity and inhibition of } \\
\text { tumor growth and metastases }\end{array}$ & {$[79]$} \\
\hline & $\begin{array}{l}\text { Doxorubicin-gold } \\
\text { nanoparticle conjugate }\end{array}$ & H1299 and YRC9 cell & Incubation & $\begin{array}{l}\text { Reduced cellular toxicity and increased } \\
\text { efficient delivery }\end{array}$ & {$[80]$} \\
\hline \multirow[t]{3}{*}{ Pancreatic } & Oncogenic Kras & Human foreskin fibroblast cell & Electroporation & reduced tumor growth and targeting KRAS & {$[65]$} \\
\hline & Oncogenic Kras & Bone marrow-derived mesenchymal stemcell & Electroporation & Reduced tumor growth and targeting KRAS & {$[81]$} \\
\hline & Doxorubicin & Macrophages cell & Incubation & Increased antitumor efficacy & {$[82]$} \\
\hline \multirow[t]{2}{*}{ Prostate } & Paclitaxel & LNCaP and PC3 cell & Incubation & Increased drug cytotoxicity & [83] \\
\hline & SPIONS & Human mesenchymal cell & Incubation & Inhibition of tumor proliferation & {$[84]$} \\
\hline \multirow[t]{7}{*}{ Glioblastoma } & $\begin{array}{l}\text { Curcumin; } \\
\text { STAT3 inhibitor }\end{array}$ & GL26 cell & Incubation & $\begin{array}{l}\text { Reduced tumor growth and increased } \\
\text { Target specificity }\end{array}$ & [85] \\
\hline & MiR-124a & Mesenchymal stem cell & Incubation & $\begin{array}{l}\text { Silence Forkhead box (FOX)A2 and reduced } \\
\text { tumor growth }\end{array}$ & {$[86]$} \\
\hline & $\begin{array}{l}\text { SiRNA; Paclitaxel or } \\
\text { doxorubicin }\end{array}$ & bEND. 3 cell & Incubation & $\begin{array}{l}\text { Increased drug cytotoxicity and crossed the } \\
\text { BBB }\end{array}$ & {$[87]$} \\
\hline & Doxorubicin, paclitaxel & Brain cell & Microinjection & Tumor growth inhibition & [87] \\
\hline & miR146b & Mesenchymal stem cell & Incubation & Inhibition of tumor proliferation & {$[88]$} \\
\hline & miR9 & Mesenchymal stem cell & Incubation & $\begin{array}{l}\text { Increase in chemosensitivity and tumor } \\
\text { regression }\end{array}$ & [89] \\
\hline & Paclitaxel & Embryonic stem cell & Incubation & $\begin{array}{l}\text { strong ability to cross the BBB and enhanced } \\
\text { targeting }\end{array}$ & {$[90]$} \\
\hline \multirow[t]{2}{*}{ Ovarian } & Cisplatin & Umbilical cord-derived macrophage cell & Sonication & $\begin{array}{l}\text { Increase in chemosensitivity and drug } \\
\text { cytotoxicity }\end{array}$ & {$[91]$} \\
\hline & miR-199a-3p & Omental fibroblasts of OC patients & Electroporation & Inhibit cell proliferation and invasion & {$[60]$} \\
\hline $\begin{array}{l}\text { Oral squamous } \\
\text { cell Carcinoma }\end{array}$ & Cabazitaxel / TRAIL & Mesenchymal stem cell & $\begin{array}{l}\text { Ultracentrifugation and } \\
\text { dialysis }\end{array}$ & Tumor growth inhibition & [92] \\
\hline \multirow[t]{3}{*}{ Hepatocellular } & miR-31,miR-451a & Plasma & Electroporation & Silence target genes and promote apoptosis & [93] \\
\hline & $\mathrm{rAAV} / \mathrm{AFP}$ & Human peripheral blood dendritic cell & Transfection & Increased drug cytotoxicity & [94] \\
\hline & miR-26a & 293T cell & Electroporation & Inhibit cell proliferation[95] & [95] \\
\hline Melanoma & Ovalbumin & Dendritic cell & Incubation & Tumor growth inhibition & [96] \\
\hline Gastric & rMETase & Immature dendritic cell & Electroporation & Tumor growth inhibition & [97] \\
\hline \multirow[t]{3}{*}{ Colorectal } & 5-FU, miR-21 & $\begin{array}{l}\text { Culture supernatants of THLG-293T or LG-293T } \\
\text { cell }\end{array}$ & Ultracentrifugation & $\begin{array}{l}\text { Enhanced the cytotoxicity and reverse drug } \\
\text { resistance }\end{array}$ & {$[98]$} \\
\hline & miR-128-3p & FHC cell & Ultracentrifugation & $\begin{array}{l}\text { Tumor growth inhibition and increase in } \\
\text { chemosensitivity }\end{array}$ & [99] \\
\hline & Doxorubicin & Human umbilical vein endothelial cell & Incubation & Tumor growth inhibition & [100] \\
\hline Leukemia & miR-21 & Plasma & Transfection & Tumor growth inhibition & [101] \\
\hline
\end{tabular}




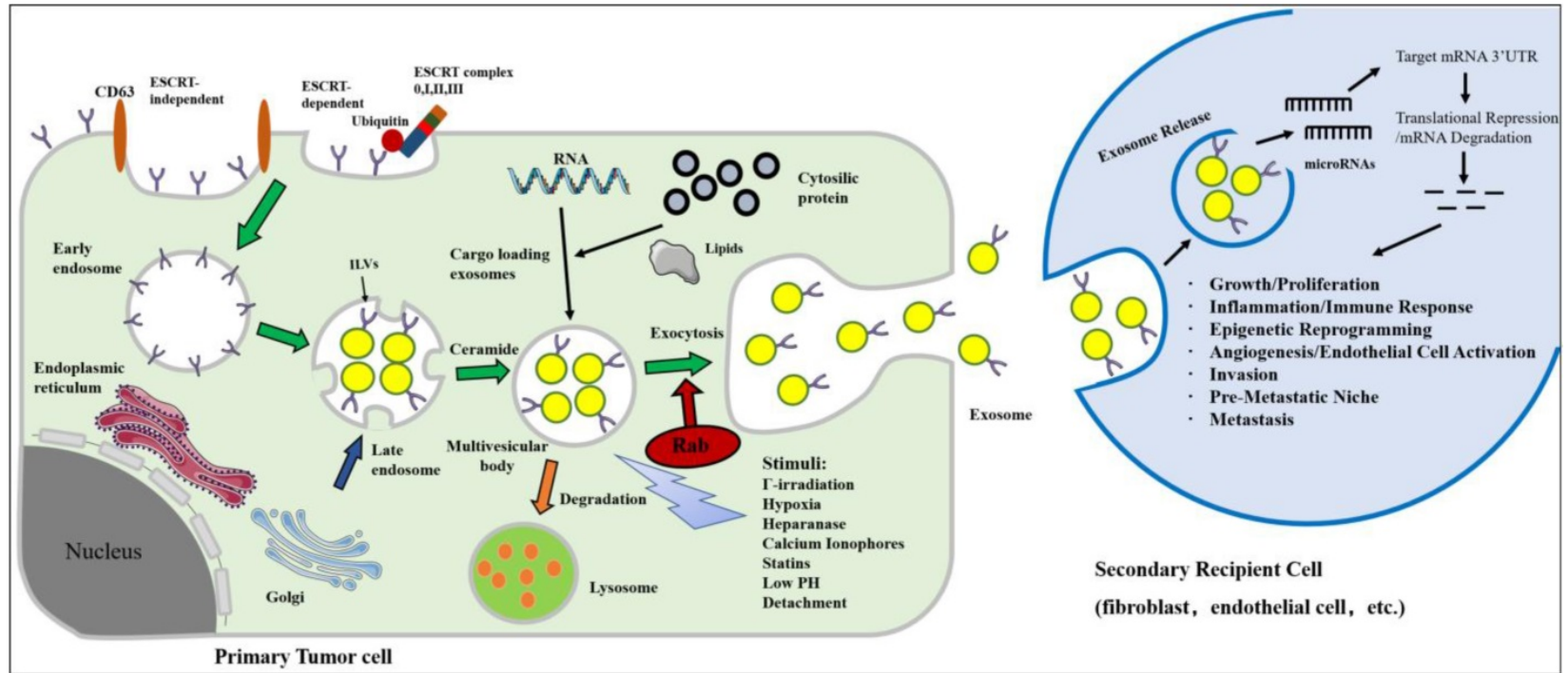

Figure 1. Biogenesis, secretion and uptake of tumor-derived exosomes in the tumor microenvironment. Inward invagination of the cell wall mediated by either ESCRT complex with the help of ubiquitin (ubiquitinated ESCRT-dependent way) or ceramide-triggered inward budding (ESCRT-independent way) in the presence of CD63. Exosomes are formed by the inward budding of the multivesicular body (MVB) membrane in the form of intraluminal vesicles (ILVs). Eventually, exosomes are secreted in exocytic MVBs following fusion of MVBs with the cell membrane, a process that depends on Rab GTPases. MVB may undergo degradation by lysosome for recycling its content. The secretion of exosomes can be stimulated by various chemical, environmental, and mechanical stimuli, such as $\Gamma$-irradiation, hypoxia, low pH, etc. Exosomes can release their microRNA cargo. The transferred microRNAs are functionally active and can regulate gene expression in recipient cells by post translationally modulating the expression of target mRNAs, leading to mRNA degradation or instability. MicroRNA dependent gene regulation can activate various processes involved in tumor development and progression.

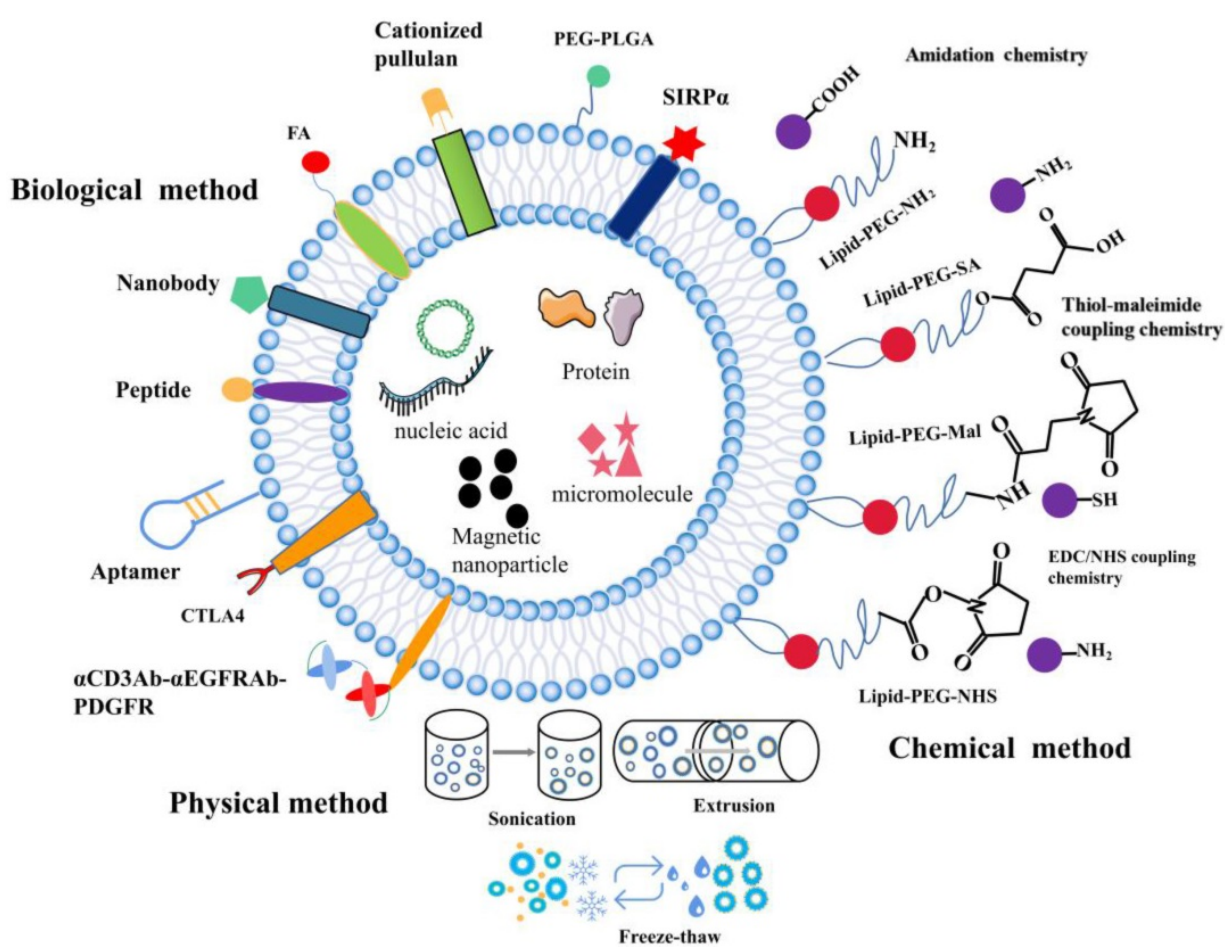

Figure 2. The surface functionalization of EVs. A schematic illustration of the physical, biological, and chemical strategies used for surface functionalization of exosomes.

Some in vivo studies have also been conducted with satisfactory results. Introduction of T7-exo and antisense miRNA oligonucleotides against miR-21 (AMO-21) via tail vein effectively introduced AMO-21 into the brain and reduced Mir-21 levels in glioblastoma in rats [62]. Downregulation of miR-21 by AMO-21 induced the expression of PDCD4 and PTEN in tumors, thereby decreased the tumor size. In a study, miR-129-3p directly inhibited the expression of SUMO-activated enzyme subunit 1 (SAE1) by targeting 3'UTR and also suppressed the zoylation of XRCC4, leading to more DNA damage in gastric cancer cells and inhibition of the proliferation, migration and invasion of gastric cancer cells [63]. The effect of exosomal encapsulated miRNAs on tumor chemical sensitivity was also investigated. Intra tumoral injection of miR-122-containing exosomes combined with sorafenib significantly reduced the 
weight and volume of tumors, indicating that exosomes from miR-122 adipose derived MSCs (AMSC) may enhance the sensitivity of human hepatocellular carcinoma (HCC) cells to chemotherapy [64]. The enhanced efficacy of engineered exosomes (iExosomes) in targeting oncogenic KRAS compared to liposomes has also been studied [65]. A key driver of common mutations in pancreatic cancer is the mutational form of GTPase KRAS. Application of a targeting method called RNA interference (RNAi) successfully inhibited tumor growth and significantly increased overall survival of mice with pancreatic cancer.

\section{Modification of EVs for targeted delivery}

The composition of EVs can be modified at the cellular level. EVs from various biological sources can be modified after isolation when cell-derived exosomes are unable to meet the requirements. While preserving the membrane integrity of exosomes, functional fluorescent tags [102], imaging probes [103], immuneactivators [104], and targeted therapeutic agents can be added [105]. Modified EV surface structures allow in vivo imaging and tracking of EVs. Although EVs have been shown to be useful in vitro anticancer drug carriers, this is not the case in vivo given their non-specific toxicity and off-target effects similar to those observed in conventional chemical drugs [106]. EVs need to be functionalized with specially designated parts to optimize their transmission properties. To improve the therapeutic functions of EVs in cancer treatment, different surface functionalization strategies have been proposed as shown in Figure 2.

\section{Surface engineering of EVs}

To further increase the functions of EVs, different surface engineering strategies have been explored, which can be roughly divided into three main approaches: physical, chemical, and biological approaches.

\section{Physical methods}

In this approach, physical approaches such as ultrasonic treatment, extrusion, and freeze-thaw are used to temporarily destroy lipid structures. Once the structures are removed, the vesicles spontaneously re-assemble into their natural structure. Sagar et al. hybridized macrophage-derived exosomes with liposomes through freeze-thaw method which allowed membrane fusion to form hybrid immune exosomes for doxorubicin treatment of breast cancer [107]. Since freezing and thawing can lead to denaturation of proteins, to avoid this, they applied extrusion method to promote membrane fusion.
Natural killer cell exosomes (NKsome) were prepared by simple liposome membrane extrusion technology [108]. The engineered NKsome successfully retained the targeted proteins associated with the NK cell membrane on its surface and showed higher affinity for cancer cells. Moreover, doxorubicin-loaded NKsome showed good homing efficiency in vivo, and its plasma retention time was extended by $18 \mathrm{~h}$ providing strong therapeutic effects. A biomimetic nanostructure (BNS) with a multimodal imaging system was designed to coat polymer nanoparticles with natural killer cell membrane (NKM), near-infrared ray (NIR) fluorescent dye and Gadolinium $(\mathrm{Gd})$ conjugated magnetic resonance imaging (MRI) contrast agent [109], which allowed in vitro and in vivo tracking [110]. The physical approach of surface functionalization allows for the simple reagent free functionalization of exosomes. Compared to biological and chemical methods, physical method does not require additional reagents or cell-based systems.

\section{Chemical methods}

Chemical approaches for surface functionalization involve direct use of chemical reagents to anchor functional parts to the surface of exosomes. The transmembrane protein portion of phospholipids or amine/carboxylate on surface of exosomes can be directly functionalized with various functional groups. Alternatively, functional phospholipids obtained from exosomes can be added to exosomes by simple incubation using a hydrophobic insertion strategy. Firstly, lipid functionalization of exosomes sealed with maleimide is achieved via the hydrophobic insertion strategy. A hydrophobic insertion strategy in which maleimide-terminated DSPE-PEG-Mal is used as a labeling probe, which is labeled with fluorescent dye containing maleimide for monitoring of cell communication [111]. Secondly, azide can be linked with cargo-conjugated dibenzobicyclooctyne (DBCO) through azide-alkyne cycloaddition. Wang et al. combined metabolic markers of newly synthesized proteins or polysaccharides/glycoproteins from secreting exosomal cells with chemically active azido groups, modified and functionalized exosomes through bioorthogonal click conjugation [112]. Lastly, copper-catalyzed azide-alkyne cycloaddition is another click chemistry strategy that can be used for surface functionalization of azide-functioning exosomes. Exosomes were chemically crosslinked with alkyne groups by carbodiimide and coupled to a model azide, fluoroazide 545. The coupling had no by-effect on the size of exosomes, nor did it alter the degree of adhesion/internalization of exosomes to 
recipient cells. This technique is superior to other exosome-labeling methods and is likely to find widespread application in exosome research [113]. These chemical methods are widely used to functionalize biomolecules because they are easy, fast and compatible with biomolecules.

\section{Biological method}

Studies show that different surface functionalization strategies can enhance targeting of exosomes by adding targeted peptides or ligands to EV surfaces. Expression of a well-characterized exosomal membrane protein (Lamp2b) on engineered exosome DCs fused to av integrin-specific iRGD peptide has been reported to promote tumor targeting [53]. EVs modified with GE11 peptide, a synthetic peptide that binds specifically to EGFR, could efficiently deliver let-7a miRNA to EGFR-expressing xenograft breast cancer tissues in mice, leading to a marked inhibition of tumor growth [114]. Besides, specific targeting molecules such as folic acid (FA), iron oxide, and aptamer have been used to modify EV. Aptamers with high affinity and specificity for their targets are often considered as substitutes for antibodies in targeted delivery. Targeted delivery of miR-21 to leukemia cells was achieved by modifying exosomes with the cholesterol-conjugated aptamer AS1411 [115]. Most tumors are angiogenic and produce exosomes, and novel strategies targeting exosome induced angiogenesis can reduce tumorigenesis [116]. Corrado et al. reported that carboxyamidotriazole orotate (CTO) targets tumor exosomes promoted IL-8 expression and cell adhesion of endothelial cells (ECs). Therefore, CTO inhibits the effects of these exosomes on ECs-chronic myelogenous leukaemia (CML) interaction and the migration of ECs, suppresses angiogenesis induced by exosomes [117]. This resulted in enhanced specific targeting of cancer cells and effective inhibition of tumor growth. Folate receptor (FR) is a glycosylphosphatidylinositol glycoprotein anchored on the cell surface, which is overexpressed in many epithelial malignancies, including ovarian, breast and lung cancers. Erastin-loaded exosomes labeled with FA were used to target triple negative breast cancer (TNBC) cells overexpressing FA receptors [71]. Tissue-specific delivery may also be achieved by loading exosomes with magnetic nanoparticles. It has been reported that exosomes anchored with cell-targeted peptides (CPPs) and TNF containing superparamagnetic iron oxide nanoparticles improve tumor targeting in external magnetic fields and inhibit tumor growth [118]. Surface-functionalization approach promotes expression of targeted cargo on exosomes surface. Apart from peptides, incorporating targeting proteins containing nanobodies to exosome surface has also been demonstrated as an attractive targeting strategy. Cheng et al. linked a rather sophisticated polypeptide composed of two single chain variable fragment $(\mathrm{scFv})$ antibodies targeting CD3 and EGFR on top of the domain of human platelet-derived growth factor (PDGFR) [55]. Here, they integrated with EVs by the EV biogenesis process to obtain surface-functionalized EVs with antibodies. Exosomes prepared from Ovalbumin (OVA)-pulsed, activated dendritic cells were modified with anti-CTLA-4 antibody to block this inhibitory molecule and to enhance the specificity of the exosomes toward $\mathrm{T}$ cells. This study provides a unique strategy to endow exosomal membranes with the function of anti-CTLA-4 antibody to synergize the efficacy of cancer vaccines and checkpoint blockade on tumors [119].

\section{Remaining concerns and future perspectives}

The extraction, isolation, and identification of tumor cell-derived EVs can be used to elucidate the mechanisms underlying tumor progression and provide potential therapeutic targets for cancer patients. Secondly, EVs can be loaded in different antitumor drugs to treat cancer. Lastly, with increasing interest in tumor immunotherapy, EV immunomodulatory properties mainly include regulating antigen presentation, immune surveillance, and immune activation. It is likely that a new approach to tumor immunotherapy will be revealed through an in-depth study of the molecular mechanisms underlying the interaction between EVs and immune cells.

As intermediates of intercellular communication, EVs have heterogeneous and pleiotropic physiological and pathological roles. However, many issues still need to be addressed before its clinical application. Due to tissue and cell specificity, not all tissues and cells express these so-called EV markers. Therefore, further investigation into how to identify EVs is a significant goal for future research. Isolation of purification specific EVs is limited by technical limitations, and specific EVs by the availability of suitable biomarkers as well as expensive techniques. It is necessary that we develop standard and highly efficient methods for EV isolation, purification, characterization, and manipulation that allow these vesicles to be successfully applied in the clinic.

\section{Conclusion}

In the field of nanofabrication, EVs have been widely applied in the diagnosis and treatment of tumors, but the clinical application of exosomes still 
faces arduous challenges. EVs play important roles in the tumor microenvironment, tumor and non-tumor tissue. Considering their good biocompatibility, tumor targeting and low immunogenicity, EVs have been proven to be potential drug carriers. However, the complex characteristics of exosomes are not thoroughly understood, it is important to comprehensively define the many subtypes of exosomes. Although there are many challenges in the costs, technical challenges, and lack of suitable biomarkers for EVs isolation and purification, more classification and loading mechanisms of exosomes can be exploited in further research to develop efficient exosome-based drug delivery systems. Moreover, EVs have shown great promise in tumor targeting, tumor immunotherapy and inhibition of tumor metastasis. In the future, in-depth research is needed to develop effective tools for efficient drug loading, targeted modification, and studying the mechanisms that drive tumor development and response to drugs. Such efforts will also generate new ideas for the diagnosis and treatment of tumors.

\section{Abbreviations}

EV: extracellular vesicle; TEXs: tumor-derived exosomes; ISEV: International Society for Extracellular Vesicles; NVs: nanovesicles; EXOs: exosomes; MVs: microvesicles; ABs: apoptotic bodies; DC: dendritic cell; MSCs: mesenchymal stem cells; mtDNA: mitochondrial DNA; DEX: dendritic cell-derived exosome; MHC class I: major histocompatibility complexes class I; NSCLC: non-small cell lung cancer; TAAs: tumor-associated antigens; DOX: Doxorubicin; EGFR: epidermal growth factor receptor; SMART-Exosome: synthetic multivalent antibodies retargeted exosomes; ELV: exosome-like vesicle; Anthos: anthocyanins; BBB: blood-brain barrier; Mir-21I: Mir-21 inhibitor oligonucleotide; HER2: human epidermal growth factor receptor-2; SAE1: SUMO-activated enzyme subunit 1; AMSC: adipose derived MSC; HCC: human hepatocellular carcinoma; iExosomes: engineered exosomes; RNAi: RNA interference; MVB: multivesicular body; ILVs: intraluminal vesicles; NKsome: Natural killer cell exosome; NKM: natural killer cell membrane; NIR: near-infrared ray; MRI: magnetic resonance imaging; DBCO: dibenzobicyclooctyne; FA: folic acid; CTO: Carboxyamidotriazole orotate; ECs: endothelial cells; FR: folate receptor; TNBC: triple negative breast cancer; CPPs: cell-targeted peptides; scFv: single chain variable fragment; PDGFR: human platelet-derived growth factor; OVA: Ovalbumin; ECs-CML: endothelial cells-chronic myelogenous leukaemia.

\section{Acknowledgments}

This work was supported by Zhejiang Provincial Natural Science Foundation of China (LY20H160008), Department of Health of Zhejiang Province (2019329972), The National Natural Science Foundation of China (82074052), Hangzhou Social Development of Scientific Research projects (20191203B17), Key projects of National Natural Science Foundation of China (81730108).

\section{Author Contributions}

Shuling Wang, Tian Xie, Qingchang Tian drafted the work organized co-author to write this review, and give final approval of the version to be published. Ruhua Luo, Mengmeng Liu, Tiantian Tan, Qian Yang, Yue Wang, Lianhui Men, Liping Zhao, Honghua Zhang accomplished the main text of manuscript. And Ruhua Luo, Mengmeng Liu, Qian Yang, Yue Wang accomplished the tables and abbreviations in the manuscript. Ruhua Luo, Tiantian Tan accomplished the figure in the manuscript.

\section{Competing Interests}

The authors have declared that no competing interest exists.

\section{References}

1. Feng RM, Zong YN, Cao SM, et al. Current cancer situation in China: good or bad news from the 2018 Global Cancer Statistics? Cancer Commun. 2019; 39: 12.

2. Dong P, Rakesh KP, Manukumar HM, et al. Innovative nano-carriers in anticancer drug delivery-a comprehensive review. Bioorganic Chemistry. 2019; 85: 325-36

3. Tian T, Zhang H-X, He C-P, et al. Surface functionalized exosomes as targeted drug delivery vehicles for cerebral ischemia therapy. Biomaterials. 2018; 150: 137-49.

4. Kim JH, Kim E, Lee MY. Exosomes as diagnostic biomarkers in cancer. Mol Cell Toxicol. 2018; 14: 113-22.

5. Zhao L, Gu C, Gan Y, et al. Exosome-mediated siRNA delivery to suppress postoperative breast cancer metastasis. J Control Release. 2020; 318: 1-15.

6. Yang Z, Zhao N, Cui J, et al. Exosomes derived from cancer stem cells of gemcitabine-resistant pancreatic cancer cells enhance drug resistance by delivering miR-210. Cellular Oncology. 2020; 43: 123-36.

7. Jeong K, Yu YJ, You JY, et al. Exosome-mediated microRNA-497 delivery for anti-cancer therapy in a microfluidic 3D lung cancer model. Lab Chip. 2020; 20: 548-57.

8. Liang G, Kan S, Zhu Y, et al. Engineered exosome-mediated delivery of functionally active miR-26a and its enhanced suppression effect in HepG2 cells. Int J Nanomedicine. 2018; 13: 585-99.

9. Théry C, Witwer KW, Aikawa E, et al. Minimal information for studies of extracellular vesicles 2018 (MISEV2018): a position statement of the International Society for Extracellular Vesicles and update of the MISEV2014 guidelines. J Extracell Vesicles. 2018; 7: 1535750.

10. Nikfarjam S, Rezaie J, Kashanchi F, et al. Dexosomes as a cell-free vaccine for cancer immunotherapy. Journal of experimental \& clinical cancer research : CR. 2020; 39: 258 .

11. Wang $\mathrm{C}$, Huang $\mathrm{X}, \mathrm{Wu} \mathrm{YJ}$, et al. Tumor Cell-associated Exosomes Robustly Elicit Anti-tumor Immune Responses through Modulating Dendritic Cell Vaccines in Lung Tumor. Int J Biol Sci. 2020; 16: 633-43.

12. Li WY, Zhu QB, Jin LY, et al. Exosomes derived from human induced pluripotent stem cell-derived neural progenitor cells protect neuronal function under ischemic conditions. Neural Regeneration Research. 2021; 16: 2064-70.

13. Skogberg G, Lundberg V, Berglund M, et al. Human thymic epithelial primary cells produce exosomes carrying tissue-restricted antigens. Immunology and Cell Biology. 2015; 93: 727-34. 
14. Akbari A, Rezaie J. Potential therapeutic application of mesenchymal stem cell-derived exosomes in SARS-CoV-2 pneumonia. Stem cell research \& therapy. 2020; 11: 356.

15. Ha D, Yang NN, Nadithe V. Exosomes as therapeutic drug carriers and delivery vehicles across biological membranes: current perspectives and future challenges. Acta Pharm Sin B. 2016; 6: 287-96.

16. $\mathrm{Xu} \mathrm{KH}$, Zhang $\mathrm{CP}, \mathrm{Du} \mathrm{TT}$, et al. Progress of exosomes in the diagnosis and treatment of lung cancer. Biomed Pharmacother. 2021; 134: 111111.

17. D'Asti E, Chennakrishnaiah S, Lee TH, et al. Extracellular Vesicles in Brain Tumor Progression. Cellular and Molecular Neurobiology. 2016; 36: 383-407.

18. Liu B, Pilarsky C. Analysis of DNA Hypermethylation in Pancreatic Cancer Using Methylation-Specific PCR and Bisulfite Sequencing. Methods Mol Biol. 2018; 1856: 269-82.

19. Akbari A, Jabbari N, Sharifi R, et al. Free and hydrogel encapsulated exosome-based therapies in regenerative medicine. Life sciences. 2020; 249: 117447.

20. Kowal J, Tkach M, Théry C. Biogenesis and secretion of exosomes. Current opinion in cell biology. 2014; 29: 116-25.

21. Hannafon $\mathrm{BN}$, Ding WQ. Intercellular communication by exosome-derived microRNAs in cancer. Int J Mol Sci. 2013; 14: 14240-69.

22. Urbanelli L, Magini A, Buratta S, et al. Signaling pathways in exosomes biogenesis, secretion and fate. Genes. 2013; 4: 152-70.

23. Baaklini I, Gonçalves CC, Lukacs GL, et al. Selective Binding of HSC70 and its Co-Chaperones to Structural Hotspots on CFTR. Sci Rep. 2020; 10: 4176.

24. Simpson RJ, Lim JW, Moritz RL, et al. Exosomes: proteomic insights and diagnostic potential. Expert review of proteomics. 2009; 6: 267-83.

25. Sharma DS, Raghuwanshi S, Kovuru N, et al. Virodhamine, an endocannabinoid, induces megakaryocyte differentiation by regulating MAPK activity and function of mitochondria. J Cell Physiol. 2021; 236: 1445-53.

26. Lian Q, Xu J, Yan S, et al. Chemotherapy-induced intestinal inflammatory responses are mediated by exosome secretion of double-strand DNA via AIM2 inflammasome activation. Cell Res. 2017; 27: 784-800.

27. Gyorgy B, Szabo TG, Pasztoi M, et al. Membrane vesicles, current state-of-the-art: emerging role of extracellular vesicles. Cell Mol Life Sci. 2011; 68: 2667-88

28. Yuan DF, Zhao YL, Banks WA, et al. Macrophage exosomes as natural nanocarriers for protein delivery to inflamed brain. Biomaterials. 2017; 142: 1-12.

29. Zhao LW, Gu CY, Gan Y, et al. Exosome-mediated siRNA delivery to suppress postoperative breast cancer metastasis. J Control Release. 2020; 318: $1-15$

30. Webb RL, Kaiser EE, Scoville SL, et al. Human Neural Stem Cell Extracellular Vesicles Improve Tissue and Functional Recovery in the Murine Thromboembolic Stroke Model. Transl Stroke Res. 2018; 9: 530-9.

31. Guo LY, Lai PL, Wang YL, et al. Extracellular vesicles derived from mesenchymal stem cells prevent skin fibrosis in the cGVHD mouse model by suppressing the activation of macrophages and B cells immune response. Int Immunopharmacol. 2020; 84: 106541.

32. $\mathrm{Li} \mathrm{YY,} \mathrm{Zhang} \mathrm{YT,} \mathrm{Li} \mathrm{Z,} \mathrm{et} \mathrm{al.} \mathrm{Exosomes} \mathrm{as} \mathrm{Carriers} \mathrm{for} \mathrm{Antitumor}$ Therapy. Acs Biomaterials Science \& Engineering. 2019; 5: 4870-81.

33. Li R, Chibbar R, Xiang J. Novel EXO-T vaccine using polyclonal CD4(+) $\mathrm{T}$ cells armed with HER2-specific exosomes for HER2-positive breast cancer. Oncotargets Ther. 2018; 11: 7089-93.

34. Xie YF, Wu J, Xu AZ, et al. Heterologous human/rat HER2-specific exosome-targeted $\mathrm{T}$ cell vaccine stimulates potent humoral and CTL responses leading to enhanced circumvention of HER2 tolerance in double transgenic HLA-A2/HER2 mice. Vaccine. 2018; 36: 1414-22.

35. Samuel M, Gabrielsson S. Personalized medicine and back-allogeneic exosomes for cancer immunotherapy. Journal of Internal Medicine. 2021; 289: 138-46.

36. Jella KK, Nasti TH, Li ZT, et al. Exosomes, Their Biogenesis and Role in Inter-Cellular Communication, Tumor Microenvironment and Cancer Immunotherapy. Vaccines. 2018; 6: 15.

37. Chen G, Huang AC, Zhang W, et al. Exosomal PD-L1 contributes to immunosuppression and is associated with anti-PD-1 response. Nature. 2018; 560: 382-6.

38. Logtenberg MEW, Scheeren FA, Schumacher TN. The CD47-SIRP alpha Immune Checkpoint. Immunity. 2020; 52: 742-52.

39. Koh E, Lee EJ, Nam GH, et al. Exosome-SIRP alpha, a CD47 blockade increases cancer cell phagocytosis. Biomaterials. 2017; 121: 121-9.

40. Wang C, Huang X, Wu Y, et al. Tumor Cell-associated Exosomes Robustly Elicit Anti-tumor Immune Responses through Modulating Dendritic Cell Vaccines in Lung Tumor. Int J Biol Sci. 2020; 16: 633-43.
41. Bol KF, Schreibelt G, Gerritsen WR, et al. Dendritic Cell-Based Immunotherapy: State of the Art and Beyond. Clin Cancer Res. 2016; 22: 1897-906.

42. Yang XY, Chen JL, Wang N, et al. Clinical use of dendritic cell-derived exosomes for hepatocellular carcinoma immunotherapy: How far we are? J Hepatol. 2018; 69: 984-6.

43. Damo M, Wilson DS, Simeoni E, et al. TLR-3 stimulation improves anti-tumor immunity elicited by dendritic cell exosome-based vaccines in a murine model of melanoma. Sci Rep-Uk. 2015;5: 17622.

44. Morse MA, Garst J, Osada T, et al. A phase I study of dexosome immunotherapy in patients with advanced non-small cell lung cancer. J Transl Med. 2005; 3: 9.

45. Besse B, Charrier M, Lapierre V, et al. Dendritic cell-derived exosomes as maintenance immunotherapy after first line chemotherapy in NSCLC. Oncoimmunology. 2016; 5: 13.

46. Pitt JM, Charrier M, Viaud S, et al. Dendritic Cell-Derived Exosomes as Immunotherapies in the Fight against Cancer. J Immunol. 2014; 193: 1006-11.

47. Morishita M, Takahashi Y, Matsumoto A, et al. Exosome-based tumor antigens-adjuvant co-delivery utilizing genetically engineered tumor cell-derived exosomes with immunostimulatory CpG DNA. Biomaterials. 2016; 111: 55-65.

48. Airola MV, Shanbhogue P, Shamseddine AA, et al. Structure of human nSMase2 reveals an interdomain allosteric activation mechanism for ceramide generation. P Natl Acad Sci USA. 2017; 114: E5549-E58.

49. Dou X-q, Wang H, Zhang J, et al. Aptamer-drug conjugate: targeted delivery of doxorubicin in a HER3 aptamer-functionalized liposomal delivery system reduces cardiotoxicity. Int J Nanomed. 2018; 13: 763-76.

50. Pulit-Prociak J, Kabat M, Wegrzyn E, et al. Encapsulation of antioxidant compounds in biopolymer micelles. Chem Eng Commun. 2020; 207: 393-412.

51. Kalaycioglu GD, Aydogan N. Layer-by-layer coated microcapsules with lipid nanodomains for dual-drug delivery. Colloids and Surfaces a-Physicochemical and Engineering Aspects. 2020; 584: 11.

52. Munagala R, Aqil F, Jeyabalan J, et al. Exosomal formulation of anthocyanidins against multiple cancer types. Cancer Lett. 2017; 393: 94-102.

53. Tian YH, Li SP, Song J, et al. A doxorubicin delivery platform using engineered natural membrane vesicle exosomes for targeted tumor therapy. Biomaterials. 2014; 35: 2383-90.

54. O'Brien K, Lowry MC, Corcoran C, et al. miR-134 in extracellular vesicles reduces triple-negative breast cancer aggression and increases drug sensitivity. Oncotarget. 2015; 6: 32774-89.

55. Cheng Q, Shi XJ, Han ML, et al. Reprogramming Exosomes as Nanoscale Controllers of Cellular Immunity. Journal of the American Chemical Society. 2018; 140: 16413-7.

56. Lin Q, Qu M, Zhou B, et al. Exosome-like nanoplatform modified with targeting ligand improves anti-cancer and anti-inflammation effects of imperialine. J Control Release. 2019; 311: 104-16.

57. Yang T, Martin P, Fogarty B, et al. Exosome Delivered Anticancer Drugs Across the Blood-Brain Barrier for Brain Cancer Therapy in Danio Rerio. Pharmaceutical Research. 2015; 32: 2003-14.

58. Johnsen KB, Gudbergsson JM, Skov MN, et al. A comprehensive overview of exosomes as drug delivery vehicles - Endogenous nanocarriers for targeted cancer therapy. Biochim Biophys Acta-Rev Cancer. 2014; 1846: 75-87.

59. Koppers-Lalic D, Hogenboom MM, Middeldorp JM, et al. Virus-modified exosomes for targeted RNA delivery; A new approach in nanomedicine. Advanced Drug Delivery Reviews. 2013; 65: 348-56.

60. Kobayashi M, Sawada K, Miyamoto M, et al. Exploring the potential of engineered exosomes as delivery systems for tumor-suppressor microRNA replacement therapy in ovarian cancer. Biochem Bioph Res Co. 2020; 527: 153-61.

61. Liang GF, Zhu YL, Ali DJ, et al. Engineered exosomes for targeted co-delivery of miR-21 inhibitor and chemotherapeutics to reverse drug resistance in colon cancer. Journal of Nanobiotechnology. 2020; 18: 15 .

62. Kim G, Kim M, Lee $Y$, et al. Systemic delivery of microRNA-21 antisense oligonucleotides to the brain using T7-peptide decorated exosomes. J Control Release. 2020; 317: 273-81.

63. Zhang MM, Jiang DL, Xie XJ, et al. miR-129-3p inhibits NHEJ pathway by targeting SAE1 and represses gastric cancer progression. Int J Clin Exp Pathol. 2019; 12: 1539-47.

64. Lou GH, Song XL, Yang F, et al. Exosomes derived from miR-122-modified adipose tissue-derived MSCs increase chemosensitivity of hepatocellular carcinoma. J Hematol Oncol. 2015; 8: 11.

65. Kamerkar S, LeBleu VS, Sugimoto $\mathrm{H}$, et al. Exosomes facilitate therapeutic targeting of oncogenic KRAS in pancreatic cancer. Nature. 2017; 546: 498-503. 
66. Haney MJ, Zhao YL, Jin YS, et al. Macrophage-Derived Extracellular Vesicles as Drug Delivery Systems for Triple Negative Breast Cancer (TNBC) Therapy. J Neuroimmune Pharm. 2020; 15: 487-500.

67. Gong CA, Tian J, Wang Z, et al. Functional exosome-mediated co-delivery of doxorubicin and hydrophobically modified microRNA 159 for triple-negative breast cancer therapy. Journal of Nanobiotechnology. 2019; 17: 18.

68. Melzer C, Rehn V, Yang YY, et al. Taxol-Loaded MSC-Derived Exosomes Provide a Therapeutic Vehicle to Target Metastatic Breast Cancer and Other Carcinoma Cells. Cancers. 2019; 11: 20.

69. Limoni SK, Moghadam MF, Moazzeni SM, et al. Engineered Exosomes for Targeted Transfer of siRNA to HER2 Positive Breast Cancer Cells. Appl Biochem Biotechnol. 2019; 187: 352-64.

70. Hadla M, Palazzolo S, Corona G, et al. Exosomes increase the therapeutic index of doxorubicin in breast and ovarian cancer mouse models. Nanomedicine. 2016; 11: 2431-41.

71. Yu MY, Gai CC, Li ZHR, et al. Targeted exosome-encapsulated erastin induced ferroptosis in triple negative breast cancer cells. Cancer Sci. 2019; 110: 3173-82.

72. Rayamajhi S, Nguyen TDT, Marasini R, et al. Macrophage-derived exosome-mimetic hybrid vesicles for tumor targeted drug delivery. Acta Biomater. 2019; 94: 482-94.

73. Aqil F, Munagala R, Jeyabalan J, et al. Exosomes for the Enhanced Tissue Bioavailability and Efficacy of Curcumin. Aaps Journal. 2017; 19: 1691-702.

74. Kim MS, Haney MJ, Zhao Y, et al. Development of exosome-encapsulated paclitaxel to overcome MDR in cancer cells. Nanomedicine-Nanotechnology Biology and Medicine. 2016; 12: 655-64.

75. Agrawal AK, Aqil F, Jeyabalan J, et al. Milk-derived exosomes for oral delivery of paclitaxel. Nanomedicine-Nanotechnology Biology and Medicine. 2017; 13: 1627-36.

76. Munagala R, Aqil F, Jeyabalan J, et al. Bovine milk-derived exosomes for drug delivery. Cancer Lett. 2016; 371: 48-61.

77. Sun DM, Zhuang XY, Grizzle W, et al. A novel nanoparticle drug delivery system: The anti-inflammatory activity of curcumin is enhanced when encapsulated in exosomes. Mol Ther. 2010; 18: 1606-14.

78. Aqil F, Kausar H, Agrawal AK, et al. Exosomal formulation enhances therapeutic response of celastrol against lung cancer. Exp Mol Pathol. 2016; 101: 12-21.

79. Kim MS, Haney MJ, Zhao YL, et al. Engineering macrophage-derived exosomes for targeted paclitaxel delivery to pulmonary metastases: in vitro and in vivo evaluations. Nanomedicine-Nanotechnology Biology and Medicine. 2018; 14: 195-204.

80. Srivastava A, Amreddy N, Babu A, et al. Nanosomes carrying doxorubicin exhibit potent anticancer activity against human lung cancer cells. Sci Rep-Uk. 2016; 6: 15.

81. Mendt M, Kamerkar S, Sugimoto H, et al. Generation and testing of clinical-grade exosomes for pancreatic cancer. Jci Insight. 2018; 3: 22.

82. Kanchanapally R, Deshmukh SK, Chavva SR, et al. Drug-loaded exosomal preparations from different cell types exhibit distinctive loading capability, yield, and antitumor efficacies: a comparative analysis. Int J Nanomed. 2019; 14: 531-41.

83. Saari H, Lazaro-Ibanez E, Viitala T, et al. Microvesicle- and exosome-mediated drug delivery enhances the cytotoxicity of Paclitaxel in autologous prostate cancer cells. J Control Release. 2015; 220: 727-37.

84. Altanerova U, Babincova M, Babinec $\mathrm{P}$, et al. Human mesenchymal stem cell-derived iron oxide exosomes allow targeted ablation of tumor cells via magnetic hyperthermia. Int J Nanomed. 2017; 12: 7923-36.

85. Zhuang XY, Xiang XY, Grizzle W, et al. Treatment of Brain Inflammatory Diseases by Delivering Exosome Encapsulated Anti-Inflammatory Drugs From the Nasal Region to the Brain (vol 19, pg 1769, 2011). Mol Ther. 2012; 20: 239-

86. Lang FM, Hossain A, Gumin J, et al. Mesenchymal stem cells as natural biofactories for exosomes carrying miR-124a in the treatment of gliomas. Neuro-Oncology. 2018; 20: 380-90.

87. Yang TZ, Martin P, Fogarty B, et al. Exosome Delivered Anticancer Drugs Across the Blood-Brain Barrier for Brain Cancer Therapy in Danio Rerio. Pharmaceutical Research. 2015; 32: 2003-14.

88. Katakowski $M$, Buller $B$, Zheng $X G$, et al. Exosomes from marrow stromal cells expressing miR-146b inhibit glioma growth. Cancer Lett. 2013; 335: 201-4.

89. Munoz JL, Bliss SA, Greco SJ, et al. Delivery of Functional Anti-miR-9 by Mesenchymal Stem Cell-derived Exosomes to Glioblastoma Multiforme Cells Conferred Chemosensitivity. Mol Ther-Nucl Acids. 2013; 2: 11.

90. Zhu QW, Ling XZ, Yang YL, et al. Embryonic Stem Cells-Derived Exosomes Endowed with Targeting Properties as Chemotherapeutics Delivery Vehicles for Glioblastoma Therapy. Adv Sci. 2019; 6: 11.
91. Zhang XH, Liu L, Tang ML, et al. The effects of umbilical cord-derived macrophage exosomes loaded with cisplatin on the growth and drug resistance of ovarian cancer cells. Drug Dev Ind Pharm. 2020; 46: 1150-62.

92. Qiu YL, Sun JM, Qiu JP, et al. Antitumor Activity of Cabazitaxel and MSC-TRAIL Derived Extracellular Vesicles in Drug-Resistant Oral Squamous Cell Carcinoma. Cancer Manag Res. 2020; 12: 10809-20.

93. Pomatto MAC, Bussolati B, D'Antico S, et al. Improved Loading of Plasma-Derived Extracellular Vesicles to Encapsulate Antitumor miRNAs. MolTher-Methods Clin Dev. 2019; 13: 133-44.

94. Li JY, Huang SL, Zhou ZF, et al. Exosomes derived from rAAV/AFP-transfected dendritic cells elicit specific $\mathrm{T}$ cell-mediated immune responses against hepatocellular carcinoma. Cancer Manag Res. 2018; 10: 4945-57.

95. Liang GF, Kan S, Zhu YL, et al.. Engineered exosome-mediated delivery of functionally active miR-26a and its enhanced suppression effect in HepG2 cells. Int J Nanomed. 2018; 13: 585-99.

96. Hiltbrunner $\mathrm{S}$, Larssen $\mathrm{P}$, Eldh $\mathrm{M}$, et al. Exosomal cancer immunotherapy is independent of MHC molecules on exosomes. Oncotarget. 2016; 7: 38707-17.

97. Xin L, Yuan YW, Liu C, et al. Preparation of Internalizing RGD-Modified Recombinant Methioninase Exosome Active Targeting Vector and Antitumor Effect Evaluation. Dig Dis Sci. 2021; 66: 1045-53.

98. Liang GF, Zhu YL, Ali DJ, et al. Engineered exosomes for targeted co-delivery of miR-21 inhibitor and chemotherapeutics to reverse drug resistance in colon cancer. Journal of Nanobiotechnology. 2020; 18: 10

99. Liu T, Zhang X, Du LT, et al. Exosome-transmitted miR-128-3p increase chemosensitivity of oxaliplatin-resistant colorectal cancer. Mol Cancer. 2019; 18: 43.

100. Jang SC, Kim OY, Yoon CM, et al. Bioinspired exosome-mimetic nanovesicles for targeted delivery of chemotherapeutics to malignant tumors. ACS nano. 2013; 7: 7698-710.

101. Huang L, Gu N, Zhang XE, et al. Light-Inducible Exosome-Based Vehicle for Endogenous RNA Loading and Delivery to Leukemia Cells. Adv Funct Mater. 2019; 29: 8.

102. Antes TJ, Middleton RC, Luther KM, et al. Targeting extracellular vesicles to injured tissue using membrane cloaking and surface display. Journal of Nanobiotechnology. 2018;16: 61.

103. Tayyaba, Rehman FU, Shaikh S, et al. In situ self-assembled Ag-Fe3O4 nanoclusters in exosomes for cancer diagnosis. J Mater Chem B. 2020; 8: 2845-55.

104. Choi ES, Song J, Kang YY, et al. Mannose-Modified Serum Exosomes for the Elevated Uptake to Murine Dendritic Cells and Lymphatic Accumulation. Macromol Biosci. 2019;19:10.

105. Cao Y, Wu TT, Zhang K, et al. Engineered Exosome-Mediated Near-Infrared-II Region V2C Quantum Dot Delivery for Nucleus-Target Low-Temperature Photothermal Therapy. Acs Nano. 2019; 13: 1499-510.

106. Chinnappan M, Srivastava A, Amreddy N, et al. Exosomes as drug delivery vehicle and contributor of resistance to anticancer drugs. Cancer Lett. 2020; 486: 18-28.

107. Sato YT, Umezaki K, Sawada S, et al. Engineering hybrid exosomes by membrane fusion with liposomes. Sci Rep-Uk. 2016; 6: 11

108. Pitchaimani A, Nguyen TDT, Aryal S. Natural killer cell membrane infused biomimetic liposomes for targeted tumor therapy. Biomaterials. 2018; 160: 124-37.

109. Pitchaimani A, Nguyen TDT, Marasini R, et al. Biomimetic Natural Killer Membrane Camouflaged Polymeric Nanoparticle for Targeted Bioimaging. Adv Funct Mater. 2019; 29: 11.

110. Nguyen TDT, Marasini R, Rayamajhi S, et al. Erythrocyte membrane concealed paramagnetic polymeric nanoparticle for contrast-enhanced magnetic resonance imaging. Nanoscale. 2020; 12: 4137-49.

111. Di HX, Zeng EZ, Zhang PJ, et al. General Approach to Engineering Extracellular Vesicles for Biomedical Analysis. Anal Chem. 2019; 91: 12752-9.

112. Wang M, Altinoglu S, Takeda YS, et al. Integrating Protein Engineering and Bioorthogonal Click Conjugation for Extracellular Vesicle Modulation and Intracellular Delivery. Plos One. 2015; 10: 12.

113. Smyth T, Petrova K, Payton NM, et al. Surface Functionalization of Exosomes Using Click Chemistry. Bioconjugate Chem. 2014; 25: 1777-84.

114. Ohno S, Takanashi M, Sudo K, et al. Systemically injected exosomes targeted to EGFR deliver antitumor microRNA to breast cancer cells. Mol Ther. 2013; 21: 185-91.

115. Huang L, Gu N, Zhang XE, et al. Light-Inducible Exosome-Based Vehicle for Endogenous RNA Loading and Delivery to Leukemia Cells. Adv Funct Mater. 2019; $29: 8$.

116. Ahmadi M, Rezaie J. Tumor cells derived-exosomes as angiogenenic agents: possible therapeutic implications. J Transl Med. 2020; 18: 249.

117. Corrado C, Flugy AM, Taverna S, et al. Carboxyamidotriazole-Orotate Inhibits the Growth of Imatinib-Resistant Chronic Myeloid Leukaemia 
Cells and Modulates Exosomes-Stimulated Angiogenesis. Plos One. 2012; p:7:13.

118. Zhuang MJ, Chen XL, Du D, et al. SPION decorated exosome delivery of TNF-alpha to cancer cell membranes through magnetism. Nanoscale. 2020; 12: 173-88.

119. Phung CD, Pham TT, Nguyen HT, et al. Anti-CTLA-4 antibody-functionalized dendritic cell-derived exosomes targeting tumor-draining lymph nodes for effective induction of antitumor T-cell responses. Acta biomaterialia. 2020; 115: 371-82. 